\title{
Unusual Presentation in a Case of Generalized Aggressive Periodontitis- A Case Report
}

\author{
Authors \\ Dr Gurpreet Singh Saini, Dr Sukhmani Hundal, Dr Guntashdeep Kaur \\ Oral and Maxillofacial Surgery, SGRD, Amritsar
}

\section{Introduction}

Peripheral giant cell granuloma (PGCG) is a localized, benign, sometimes aggressive hyperplastic reactive lesion of oral cavity. Jaffe in 1953 proposed the term"giant-cell reparative granuloma" for such lesions occurring in the jaws while Berner \& Cahn in 1954 used the term "peripheral giant cell reparative granuloma" for the peripheral lesions ${ }^{1,2}$. They believed that these lesions were not neoplasms but represented as a local reparative reaction. But as the clinical behavior of most of these lesions have been inconsistent with the reparative process, thus this nomenclature has been omitted these days.

PGCG is thought to originate from the interdental tissue (periosteum or periodontal ligament) following local irritation. Periodontal disease with varying amount of subgingival plaque and calculus too act as an important local irritant factor $^{2,3,4}$. Rapidly Progressing Periodontitis (RPP) is usually seen in young adults with a female predilection.It is a multifactorial disease and mixed factors as oral flora, immunological response of patient and environment decide the severity, course and onset of the disease ${ }^{5,6,7}$.

We present here a case and management of a young female suffering from Rapidly Progressing Periodontitis with complete destruction of the alveolar bone in both the jaws with a huge PGCG in left mandibular body region.

\section{Case Report}

A 20 year female reported to Oral and Maxillofacial Department with the chief complaint of swelling in her lower jaw for the past three years with spontaneous exfoliation of few teeth andgeneralized mobility in the rest of her teeth. Intraoral examination revealed very poor oral hygiene with plaque, calculus and debris, almost floating maxillary and mandibular teeth and generalized gingival hyperplasia with bleeding on probing. Intraoral examination revealed the presence of an irregular, bluish-red lesion in the left body region of her lower jaw. The lesion extended from 31 up till distal of 37 with an approximate antero-posterior dimension of $4 \mathrm{cms}$ and a maximum buccolingual dimension of $3.5 \mathrm{~cm}$ which slowly increased to its present size (fig). The overlying mucosa was erythematous and inflamed. It was firm in consistency with a sessile base. The lesion elicited bleeding on probing. The teeth present had grade III mobility with more than $6 \mathrm{~mm}$ deep pockets. Submental and bilateral submandibular lymph nodes were tender. The OPG revealed excessive alveolar bone resorption with scooped out 
appearance in bilateral lower posterior region with multiple missing teeth in both the jaws with only basal bone remaining (fig). CECT of facial bones revealed ill-defined enhanced lesion in relation to alveolar process of left mandible causing bone destruction (fig).

Patient was referred to periodontology department for opinion regarding her gingival status. The plaque and unstimulated saliva sample were taken and sent for the microbiological assessment. Sample collected was immediately spread over blood agar plate and divided into three parts and each part spread over blood agar plate, tripticase soya agar supplemented with 5\% blood and thioglycolate broth for aerobic, anerobic and gram staining respectively.

Routine blood and urine analysis, hormonal assay, $\mathrm{Ca}^{+}, \mathrm{PO}_{4}{ }^{-}$and parathyroid levels were done and found to be within normal limits. The incisional biopsy was done for the lesion in the mandible under local anesthesia. Based on her periodontal examination, OPG, plaque and microbiological examinationshe was diagnosed with RPP and was advised extraction of all her remaining teeth. The lesion in the mandible was histologically proved to be PGCG. Histopathology revealed immature fibrous tissue containing a mixture of mononuclear cells and multinucleated giant cells showing varying numbers of pale vesicular nuclei, evenly distributed in connective tissue. The overlying epithelium of stratified squamous epithelium was ulcerated at places (fig). Under aseptic conditions under general anesthesia, the lesion was excised completely from its base and all remaining teeth in both jaws were extracted. The sockets were curetted with removal of all the granulation tissue, debris and calculus. Primary closure was attempted and healing was uneventful.

Future treatment planning for prosthetic rehabilitation included implant supported over denture in mandible and complete denture in maxilla. Patient is under follow up for the last 3 years with no similar complaints.

\section{Discussion}

PGCG is a relatively common, non-neoplastic, lesion of oral cavity developing mainly in relation to gingiva and alveolar mucosa both in dentate and non-dentate region. These are most probably inflammatory reactive lesions developing in response to chronic irritation acting on the connective tissue of periosteum, gingiva or periodontal ligament ${ }^{2,3,4,8}$. It effects a wide age ranged from 6to 88 years but most commonly seen in $4^{\text {th }}$ to $6^{\text {th }}$ decade of life with a slight female predelication, while Lester et al 2014 found an almost equal sex distribution ${ }^{3}$. Posterior mandible as compared to maxilla is found to be more affected. Comparatively, lesions present in the anterior/esthetic region are smaller in size and reported early ${ }^{8}$.Authors have proposed anterior and posterior region till first molars is more prone to PGCG keeping in view the transition from deciduous to permanent dentition occurs here due to osteolytic activity, but this hypothesis cannot be related to lesions occurring posterior to permanent molars ${ }^{9}$. In contrast Motamedi et al found no significant difference in the site of occurance of lesion ${ }^{4}$.

The reported size of PGCG ranges from as small as $0.5 \mathrm{cms}$ to large fungating growths of $7 \mathrm{cms}$ with superficial ulcerations $s^{2,3,8,10}$. The size of PGCG lesion in our case was $4 \times 3.5 \mathrm{~cm}$. Lack of awareness about oral hygiene and negligence with continous irritation from plaque and calculus might have led to such a huge size.

Lesions are mostly smooth, pedunculated sometimes sessile proliferations with a soft tofirm consistency. They usually appear as red to purplish though pigmented lesions too are reported. It usually presents as painless swellingexcept if secondarily infected or develop ulcerations due to repeated trauma $a^{3,4,10}$. As it is a soft tissue lesion it rarely affects the underlying bone though long standing and large lesions can lead to superficial erosion which is radiographically evident ${ }^{3,12}$. The differential diagnosis based on the clinical features included pyogenic granuloma, peripheral ossifying 
fibroma, peripheral ameloblastoma and Langerhans histio cystosis which were ruled out after the histopathological examination.

The etiology of PGCG is not yet clear, but chronic irritation due to ill-fitting dentures, prosthesis, sharp restorations, subgingival and supragingival plaque and calculus play a major role ${ }^{3,4,10,11}$. It also has been documented in relation to dental implants and hyperthyroidism ${ }^{3,13}$. In our patient, supragingival plaque and calculus were the most probable causes which acted as chronic irritants leading to development of PGCG. Moreover, she was diagnosed as a patient of RPP on the basis of her clinical, periodontal, radiological and microbiologic status in both her jaw.

RPP usually presents at puberty to 35 years of age with a female predilection with $83 \%$ of patients with functional defects in neutrophils or monocytes. The gingival lesions are generalized and inflammed affecting most of the teeth without any consistent pattern of distribution. It presents with acute and remission phase after appropriate antibiotic coverage, oral prophylaxis and root planning procedures. RPP may show generalized bone destruction ranging from mild crestal bone resorption to severe extensive alveolar bone destruction depending on the severity and duration of disease. Local plaque irritation produces unusual gingival enlargements deepening the gingival pockets leading the subgingival bacteria to colonize and over grow which result in rapid alveolar bone loss $5,6,7$. Spontaneous teeth exfoliation has been documented in patients with severe bone loss ${ }^{5}$. In our case patient too complained of spontaneous exfoliation of teeth. Gram staining in the microbiological assessment done in our case showed the presence of gram positive cocci and gram negative bacilli. In the aerobic culture, streptococcus viridans was predominantly obtained after 24 hours of incubation at $37^{\circ} \mathrm{c}$ while on anerobic culture, Porphyromonas gingivalis was followed by Provettala, Peptostreptococci and Capnocytophaga after 72 hours of incubation at $37^{\circ} \mathrm{c}$. Based on the culture report patient was put on appropriate antibiotic coverage prior to surgical intervention. Significant levels of antibodies to P.gingivalis, F.nucleatum, Streptococcus inter medius, Genellamorbillorum, Actinobacillus acetinomyceten comitans (AA) have been reported in literature in serum of patients suffering from RPP. Porphyromonas gingivalis and AA also have an indispensable role in eliciting an aggressive aggravated host response depending upon the genetic, immunologic and envoirnmental profile of the patient ${ }^{6}$.

Treatment modalities for RPP include root planning with complete oral prophylaxis and appropriate antibiotic coverage to decrease the subsequent acute bone resorption and thus lead into the quiesent phase and extraction of hopeless teeth, which was followed in our case $e^{5,6,7}$.

Complete surgical excision of lesion from its base along with the removal of local irritants is the treatment of choice to minimize the recurrence in case of PGCG. Recurrence reported is very minimal $4-17.5 \%$ which could be due to incomplete removal ${ }^{3,4,10,11}$. Usage of Lasers for resection of PGCG causes less bleeding, enhances postoperative patient compliance but is contraindicated in cases where through curettage is necessary especially when lesions are close to the bone ${ }^{14}$. Sclerotherapy using ethanolamine oleate (EO) has been tried and has given excellent results in treatment of PGCG as safe, effective, inexpensive with speedy results in pendunculated lesions and a low recurrence rate. It acts as an irritant when injected intralesionally producing an inflammatory response and fibrosis of the endothelium of the blood vessels of the lesion ${ }^{10}$. To conclude, PGCG and RPP are not uncommon entities encountered, but general oral health awareness, timely referral, early diagnosis and intervention can decrease the destruction process in young patients.

\section{Bibliography}

1. Jaffe HL. Giant cell reparative granuloma, traumatic bone cyst, and fibrous(fibrous 
osseous) dysplasia of the jaw bones. Oral surg Oral med Oral pathol 1953; 6: 159175.

2. Bernier JL and Cahn LR: Peripheral giant cell reparative granuloma. JADA 1954: 49;141-148.

3. Lester SR ,Cordel KG, Rosebush MS, Palaiologou AA, ManeyP.Peripheral giant cell granulomas: a series of 279 cases. Oral surg Oral med Oral pathol Oral radiol 2014; 118: 475-482.

4. Motamedi MHK, Jafari SM, Lassemi E, Abbas FM, Khalifeh S, Eshkevari PS. Peripheral and giant cell granulomas of the jaws: A demographic study. Oral surg Oral med Oral pathol Oral radiolEndol 2007; 103:e39-e43.

5. Page RC, Altman LC, Ebersole JL, Vandesteen GE, Dahlberg WH, Williams BL, Osterberg SK. Rapidly Progressive Periodontitis. A distinct clinical condition.J Periodontal April 1983: 197209.

6. Kamma JJ, Nakou M, Manti FA. Microbiota of Rapidly Progressive Periodontitis Lesions in association with clinical parameters. J Periodontol Nov 1994. 1073-1078.

7. Palcanis KG, Wolfe B, McClung JF, Elzay RP. Rapdily Progressive Periodontitis: J Periodontol June 1986, 378-382.

8. Gharbyan AZ, Assaf M. Management of a Peripheral Giant cell granuloma in the esthetic area of upper jaw: A case report. International Journal of Surgery Case reports 5(2014) 779-782.

9. Cooke BED. Giant cell epulis: Histogenesis and natural history. Br Dent J.1952: 93: 12-16.

10. Ahmed WS. Efficacy of ethanolamine oleatesclerotherapyin treatment of peripheral giant cell granuloma. J Oral Maxillofac : 1-7, 2016.
11. Katsikeris N, Angelopoulou KA, Angelopoulos AP. Peripheral giant cell granuloma. Clinicopathologic study of 224 new cases and review of 956 reported cases.Int J. Oral Maxillofac Surg.1988; 17:94-99.

12. Wood NK, Goaz PW: Differential diagnosis of oral and maxillofacial lesions (ed5). St Louis, MO, Mosby, 1997,141143.

13. Choi C, Terzian E, Schneider R, Trochessel DA. Peripheral giant cell granuloma associated with hyperthyroidism secondary to end stage renal disease: A case report. J Oral MaxillofacSurg 66: 1063-1066,2008.

14. Chaparro AV, Berini AL, Escado G: Peripheral giant cell granuloma: A report of 5 cases and review of literature. Med Oral Pathol Oral Cir Bucal 10: 48, 2005. 